\title{
Antonio da Besana à Lucerne. Un représentant des ducs de Milan à la diète confédérée (1458-1468)
}

\section{Aureliano Martini}

\section{Q OpenEdition}

\section{Journals}

Édition électronique

URL : http://journals.openedition.org/edl/255

DOI : $10.4000 /$ edl. 255

ISSN : 2296-5084

Éditeur

Université de Lausanne

\section{Édition imprimée}

Date de publication : 15 septembre 2010

Pagination : 81-98

ISBN : 978-2-940331-23-9

ISSN : 0014-2026

\section{Référence électronique}

Aureliano Martini, «Antonio da Besana à Lucerne. Un représentant des ducs de Milan à la diète confédérée (1458-1468) », Études de lettres [En ligne], 3 | 2010, mis en ligne le 15 septembre 2013, consulté le 22 décembre 2020. URL : http://journals.openedition.org/edl/255 ; DOI : https://doi.org/ 10.4000/edl.255

\section{(c) Études de lettres}




\section{ANTONIO DA BESANA À LUCERNE \\ UN REPRÉSENTANT DES DUCS DE MILAN À LA DIÈTE CONFÉDÉRÉE (1458-1468)}

Entre 1458 et 1468, Francesco Sforza nomme plusieurs fois Antonio da Besana orateur du duché de Milan auprès de la diète confédérée. Son expérience administrative avait déjà permis à ce personnage de se rapprocher du milieu et des problématiques liés aux marchands suisses qui visitaient les foires lombardes. Ainsi, la conduite des pourparlers a pu bénéficier de sa profonde connaissance des sujets de débat et de l'existence d'un véritable réseau de contacts dans les milieux commerciaux et politiques des cantons. L'analyse de ses compétences, de son action auprès des Cantons et de ses liens personnels permet enfin de montrer l'important apport de l'orateur aux relations diplomatiques entre le duché de Milan et la Confédération suisse.

Dans le cadre très vaste des rapports diplomatiques du duché de Milan, le cas des relations avec la Confédération suisse est tout à fait intéressant. Axés sur un nombre très limité de sujets, ces débats permettent de retracer l'action des acteurs, leurs liens sociaux et surtout les stratégies qui ont été élaborées et mises en œuvre. Plus précisément, les négociations pour le renouvellement des accords de paix et d'alliance qui occupent l'administration milanaise entre 1458 et 1468 offrent à l'historien la possibilité de mettre en évidence le profil des personnages qui ont travaillé à l'établissement et à la médiation des traités. En effet, si bien d'autres historiens ont analysé le contenu et l'évolution des accords diplomatiques, les structures et les réseaux sociaux qui ont permis - ou mis en difficulté la poursuite des discussions ont été moins mis en évidence ${ }^{1}$.

I. Pour l'analyse des phases qui ont porté à la signature du Gande Capitolato di Milano, traité qui est ici en jeu, cf. G. Chiesi, Il Medioevo nelle carte, p. 247-252 
Les relations entre le duché et les Suisses au $\mathrm{XV}^{\mathrm{e}}$ siècle frappent par la continuité des sujets traités, problèmes sur lesquels la diplomatie éprouve une difficulté visible à trouver une solution définitive. Ces grands thèmes - exemption des taxes de passage, facilités juridiques et cession temporaire du domaine sur la Vallée de la Léventine au canton d'Uri - apparaissent déjà dans les accords signés par Filippo Maria Visconti en 1426 et 1441. En 1450, Francesco Sforza avait enfin très vite signé un renouvellement des conditions souscrites par son beau-père, renvoyant les débats avec les Suisses à une époque peut-être moins dangereuse pour le nouveau duc ${ }^{2}$.

En 1458, le duc de Milan charge Antonio Riboldi da Besana d'une première mission diplomatique auprès de la diète confédérée ${ }^{3}$. Cette

et passim; L. Moroni Stampa, «Francesco I Sforza e gli Svizzeri (1450-1466)»; F. M. Vaglienti, " "Per dicta pace realegrati”: le trattative diplomatiche tra la Confederazione Elvetica e il duca Galeazzo M. Sforza per il rinnovo del Capitolato, l'investitura della Leventina e la cessione della Val Formazza (1466-1469)»; A. Martini, «Dialogo e compromessi: trattative diplomatiche sulla via del San Gottardo». Dans cette contribution nous ferons largement référence aux documents édités dans les volumes Ticino Ducale (par la suite TD), ouvrages qui constituent aujourd'hui un outil fondamental pour toute recherche sur les relations entre les Sforza et la Confédération suisse.

2. Dans ce traité (TD I, no 20) Francesco Sforza confirme l'exonération des péages et de toute sorte d'imposition pour les marchands et les marchandises confédérées jusqu'aux portes de Milan. Le duc accepte aussi de permettre aux Suisses de porter leurs armes (exception faite pour les villes fortifiées). Cet avantage, expressément conçu pour favoriser les intérêts suisses dans le milanais, fait pendant à un deuxième privilège grâce auquel ils peuvent demander que les causes les concernant soient jugées par giustizia sommaria, un type de procès très expéditif dans lequel le magistrat décide les peines tout de suite après avoir établi les faits (Cf. aussi G. Chiesi, «Venire cum equis ad partes Lumbardie: mercanti confederati nella seconda metà del XV secolo»). Enfin, le duc de Milan confirme au canton d'Uri la possession du fief de la Vallée de la Léventine à titre de gage contre la promesse de remboursement de 2000 ducats dans un délai de six ans. Cette disposition revêt une très grande importance dans les débats car elle définit la position des représentants d'Uri à la diète car ses autorités considèrent définitif le passage de ce territoire à la souveraineté suisse, tandis que pour l'administration milanaise la situation est beaucoup plus compliquée: au niveau juridique, le duc est seulement administrateur des droits sur la Léventine qui reste propriété du chapitre de l'Eglise majeure. L'attitude des représentants ducaux vis-à-vis de la Léventine détermine à elle seule si le waldstätte et ses partisans sont favorables ou non aux pourparlers.

3. "Illustrissimus et excellentissimus dominus dominus Franciscus Sforcia, dux Mediolani \&c., [...], volens predictas pactiones et conventiones implere et exequi, voluntarie, sponte et ex certa scientia et non aliquo errore iuris aut facti ductus, et alias 
première négociation, liée au rachat des droits dans la Vallée de la Léventine, est la première étape d'une longue période de débats durant laquelle Francesco Sforza, par l'intermédiaire de son orateur, cherchera à instaurer un nouveau dialogue avec les Suisses, à renouveler les alliances et les accords de paix et surtout à faire face aux engagements souscrits par son prédécesseur. L’ambassadeur, véritable négociateur entre les deux puissances, affrontera les débats fort de l'appui du duc et d'une extrême compétence dans les domaines liés aux Suisses: par son action, il impose une ligne diplomatique très marquée par son charisme et ses liens avec le monde confédéré. Pour mieux comprendre le rôle de cet ambassadeur, il faut ainsi analyser les différents aspects des choix stratégiques qu'il a pu mettre en pratique pendant ses missions, notamment le choix des lieux de débats, les moyens de communication avec le duc, les modalités de négociations et les réseaux utilisés.

\section{Antonio da Besana entre Bellinzone et Lucerne}

En 1450, après avoir pris le contrôle du duché de Milan, Francesco Sforza nomme Antonio da Besana podestà de Bellinzone, dernier bourg fortifié sur la route entre Milan et le col du Saint-Gothard, office qui sera vite doublé par celui de commissaire ducal ${ }^{4}$. Il met ainsi à profit sa formation de juriste et acquiert une certaine popularité: en 1452, le Petit conseil demande au duc de prolonger son mandat, mais sans succès. Antonio da Besana n'abandonne cependant pas Bellinzone et exerce l'office d'advocatus fiscalis (1454), de lieutenant du podestà (1457) et enfin à nouveau celui de podestà e commissario $(1458-1461)^{5}$.

omnibus modo, iure, via et forma quibus melius potuit et potest, fecit, constituit, creavit et solempniter ordinavit ac facit, constituit, creat et solempniter ordinat egregium et sapientem iuris utriusque doctorem dominum Antonio de Bexana suum certum, verum, legiptimum et indubitatum missum, nuncium, actorem et procuratorem specialem [...]» TD I, no 932.

4. Ibid., $\mathrm{n}^{\mathrm{o}} 9$ et $\mathrm{n}^{\mathrm{o}} 111$.

5. Francesco Sforza lui confie aussi l'office de podestà de Canobbio (1465) et de vice-podestà de Pavie (1468). Après ses missions diplomatiques, il occupera la charge de vicario e sindacatore generale (1468-1471) et de maestro delle entrate straordinarie (1472). 
Cette permanence du duché dans les districts septentrionaux lui permet d'acquérir une vaste connaissance des sujets propres aux relations avec les Suisses. Dans cette ville, première étape dans les itinéraires des marchands suisses vers Milan (notamment au moment de la foire locale), Antonio da Besana a pu connaître un certain nombre de Confédérés, dont quelques-uns vont aussi être impliqués dans les négociations diplomatiques. Pendant la période où il exerçait l'office de podestà, il réussit non seulement à acquérir une expérience directe des difficultés posées par l'arbitrage juridique mais, probablement, il parvient également à distribuer les premières faveurs qui lui ont garanti une si bonne réputation dans les territoires suisses ${ }^{6}$. A Bellinzone, il a même eu l'occasion d'apprécier les problématiques liées à la présence uranaise dans la Vallée de la Léventine, tout comme le sentiment de leurs sujets et des autorités locales.

En mai 1458, Francesco Sforza confie à Antonio da Besana une mission diplomatique auprès de la Ligue de Coire, où il doit agir en faveur du comte Enrico de Sacco, seigneur de Mesocco ${ }^{7}$. Cette mission lui permet de mettre à profit son expérience dans le but de convaincre les autorités de la Ligue de rendre à l'allié du duc certains territoires dans le Val Mesolcina. Le succès de cette mission pourrait bien être la raison principale expliquant qu'on l'ait choisi pour les missions auprès des Suisses. Dès 1458, il sera en effet chargé de plusieurs missions diplomatiques auprès de la diète suisse, instaurant avec le duc Francesco Sforza un rapport de confiance croissant ${ }^{8}$.

Pendant son séjour dans les territoires confédérés, Antonio da Besana choisit de s'installer dans la ville de Lucerne où il résida notamment entre septembre et décembre 1462, septembre 1463 et janvier 1464 ,

6. TD III, $\mathrm{n}^{\mathrm{o}} 69$.

7. $T D \mathrm{I}, \mathrm{n}^{\circ} 902$ et $\mathrm{n}^{\circ} 905$.

8. Le ton et les registres linguistiques utilisés dans les rapports entre l'orateur et le duc, mais aussi entre l'administration ducale et les structures confédérées à propos d'Antonio da Besana, semblent montrer une évidente dé-formalisation et - pourrait-on dire - familiarité. Ainsi, le dernier message de présentation d'Antonio da Besana à la diète est une simple lettre: "Mittimus ad dominationes vestras egregium et sapientem legumdoctorem dominum Antonium de Besana, oratorem nostrum dilectissimum, harum exhibitorem, pro re quam ab illo coram latius intelligetis. Hortamur igitur et rogamus ipsas magnificentias vestras ut eidem oratori nostro in omnibus que nomine nostro refert amplam indubitamque fidem ceu persone nostre proprie adhibere velint.» (Ibid., no 2026). Cf. aussi Ibid., n ${ }^{\circ} 1424, \mathrm{n}^{\circ} 1546$ et no 2026. 
ainsi qu'entre avril 1466 et août 1467. Les raisons qui ont poussé l'orateur à faire ce choix sont très simples : à Lucerne se trouvent un grand nombre de marchands et de personnalités politiques qui ont investi dans le commerce avec Milan. Ces hommes, qui soutiennent toujours l'orateur pendant son séjour, constituent le noyau principal du réseau filo-milanais au sein des Confédérés. Il serait exagéré d'affirmer que ces personnes agissent dans l'intérêt du duc; plus probablement, leurs intérêts convergent avec ceux de Milan 9 .

En tant que représentant du duc de Milan, Antonio da Besana doit entretenir dans la ville un niveau de vie digne d'un bourgeois lucernois. Son arrivée est précédée par des lettres de présentation et de recommandation ${ }^{10}$. Il dispose d'une résidence où il invite à plusieurs reprises les autorités lucernoises à souper ou à dîner ${ }^{11}$. L'orateur la définit $c a x a$, mais il est difficile de préciser s'il s'agit d'une maison de sa propriété, d'un bâtiment mis à disposition par les partisans du duc de Milan à Lucerne ou bien s'il nomme ainsi une simple auberge ${ }^{12}$. Il est accompagné par un

9. $T D$ II, $\mathrm{n}^{\circ} 60$.

Io. «Amice noster carissime [Henrico Hunvil]. Nos [Franciscus Sfortia] ambigimus vos multotiens intellexisse quam bonam de vobis relationem apud nos semper fecerit dominus Antonius de Bexana, doctor et dilectus civis huius urbis, et quantum illi magnifice Lige simul et patrie vestre affectus sit et ut illos vestris et compatriotis qui sibi eum debere pretendebant de sorte contentos reddiderit et in omnibus etiam debitum facere paratis sit. Commendamus igitur ipsum vobis et dignum arbitramur et rogamus quood et vos in concernentibus honorem et commodum suum ea presertim in causa sibio propitius sit, quod nobilitatis animi et gratitudinis vestre simul ac acceptum nobis erit, in omnibus paratis amicicie vestre beneplacitis.» (TD I, $\mathrm{n}^{\circ} 1423$ ).

II. "Ogi sono stati a disnare a caxa mia con mi quisti signori Lucernexi, cioè li sculteti et li megliori del loro conciglio secreto." (Ibid., $\mathrm{n}^{\circ}$ 1607). Ces invitations peuvent aussi être très fastueuses: «l'aviso [domine Cicho Sumonete] che fere ogni giorno me convene havere a caxa ad mangiare con mi qualche uno de quisti signori [...]. M'è convenuto sempre pagare el mangiarre in decuplo et multo ultra, però che, fornito el disnare o la cena, me bisogniava ogni volta, se non voleva recevere vergogna, presentare lì, mo' piú mo' manco, segondo la dignità del logo, il che anche me ha cavato bona soma de dinare de borsa. Et anche de questo non sono stato se no' alegro et contentissimo sopra modo per l'honore grandissimo de' nostro illustrissimo signore, purché gli fusse dove me podesse sostenire \&c. Non dico niente de' piferi, de' trombeti et de li servitori et donzeli de li signori, a' quali honorevelmente m'è convenuto presentare a questa festa \&c.» (Ibid., no 1614).

I2. Lors d'un séjour à Zurich (décembre 1466 - avril 1467), Antonio da Besana écrit une lettre à Milan dans laquelle il réclame de l'argent et souligne qu'il paye pour sa casa 4 florins du Rhin chaque mois. Vu le prolongement de son déplacement dans cette 
petit nombre de courtisans qui comprend son chancelier, son fils et cinq autres familiers ${ }^{13}$. Malgré la provision de chacun d'entre eux et le salaire mensuel de l'orateur qui se monte à 25 ducats, les frais de fonctionnement de cette ambassade restent véritablement très élevés. En 1467, au moment de faire face aux dettes accumulées au cours de sa mission, il demande à Milan d'envoyer la somme de presque 900 ducats ${ }^{14}$.

\section{Difficultés et stratégies des négociations}

\subsection{Aller au-delà du Saint-Gothard}

Le voyage de l'ambassadeur commence généralement à Milan, par un colloque avec le duc ou avec ses conseillers ${ }^{15}$. Par la suite, l'administration fournit à l'orateur une lettre dans laquelle elle explique les détails de la mission ${ }^{16}$, les lettres de présentation ${ }^{17}$ et de libre passage à travers le duché ${ }^{18}$. La première étape du voyage est Bellinzone, d'où l'orateur part pour Altdorf, puis pour Lucerne. Malgré les documents de passage donnés à l'orateur et à ses collaborateurs, la route n'est pas parfaitement sûre. Les agissements des hommes d'Uri, influencés par la conduite des négociations, provoquent parfois des troubles ${ }^{19}$ au cours desquels, en 1466, le chancelier d'Antonio da Besana est gravement blessé sur la route pour Altdorf ${ }^{20}$. Les difficultés ne se posent toutefois pas seulement sur le territoire suisse. Quelques collaborateurs ont aussi été retenus par les officiers milanais; ce fut le cas, par exemple, d'un émissaire qui a été emprisonné en 1466 par Bartolomeo Caìmi, commissaire de Bellinzone ${ }^{21}$.

ville, il est cependant impossible de savoir s'il se réfère au logement de Lucerne ou bien à celui de Zurich (TD II, no 219).

I3. Ibid.

I4. Ibid., no 428 et no 432 .

I5. TD I, no 1987.

16. Ibid., $\mathrm{n}^{\circ} 932$ et $\mathrm{n}^{\circ} 1467$.

17. Ibid., $\mathrm{n}^{\circ} 1424$ et $\mathrm{n}^{\circ} 1546$.

I8. Ibid., $\mathrm{n}^{\mathrm{o}} 1425, \mathrm{n}^{\mathrm{o}} 1545$ et $\mathrm{n}^{\mathrm{o}} 1583$.

I9. $T D$ II, $\mathrm{n}^{\circ} 176$ et $\mathrm{n}^{\circ} 256$.

20. Ibid., no 190 .

2I. Ibid., $\mathrm{n}^{\mathrm{O}} 47$. 
Ces difficultés ont aussi une influence sur les échanges épistolaires entre le duc et son orateur. Avant tout, une certaine discrétion doit être garantie sur une partie du texte, justifiant l'utilisation d'un système de cryptage selon une substitution littérale ${ }^{22}$. Il faut également considérer le délai temporel entre l'envoi et la réception du message: pour faire face à ce retard et garantir une certaine souplesse à ses stratégies, Antonio da Besana suggère au duc d'envoyer toujours deux missives: la première publique, avec des intentions exprimées d'une façon générale et ambiguë; la deuxième secrète, avec les véritables instructions pour l'orateur ${ }^{23}$. De même, l'ambassadeur répondra avec deux lettres: une pour le duc et l'autre pour son secrétaire Cicco Simonetta, celle-ci avec tous les détails qui méritent d'être transmis à Milan.

\subsection{Débats à la diète}

L’appréciation du système diplomatique suisse passe nécessairement par la compréhension du fait que la diète confédérée n’a aucun pouvoir au sens strict: elle est seulement un lieu de gestion collégiale et les décisions sont toujours prises par les autorités cantonales ${ }^{24}$. Ainsi, même si la signature des traités advient généralement à Milan, ceux-ci doivent, par la suite, être approuvés par chaque canton. Cette démarche très complexe irrite à plusieurs reprises l'administration ducale et l'orateur doit intervenir pour rappeler que ces retards dépendent du système de gouvernement suisse. Antonio da Besana a très bien compris ce fonctionnement et il a bien su conduire les débats non seulement au sein de la diète, mais aussi dans les Conseils des villes - par exemple à Berne ou à Altdorf ${ }^{25}$ - et dans les milieux sociaux des cantons, tout comme l'exemple des dîners donnés dans sa caxa l'illustre bien.

22. L. Cerioni, La diplomazia sforzesca nella seconda metà del Quattrocento e i suoi cifrari segreti. Le chiffraire concernant Antonio da Besana est consultable à la page $37 \mathrm{v}$ du deuxième volume.

23. «[La signoria vosta] me pare che la scriva per ogni modo due litere, l'una de quale se posa fare qualche demonstratione con qualche uno de quisti contraria o diversa a la vera intencione ne la quale vostra illustrissima signoria parla o scrive da foleto [...]. L'altra poy ne la quale distinctamente et chiaramente me sia dato ad intendere l'intentione d'essa illustrissima signoria vostra.» (TD I, no 1595).

24. M. Jucker, Gesandte, Schreiber, Akten, p. 76-77 et 188-194.

25. TD I, no $1604 ; T D$ II, $\mathrm{n}^{\circ} 196$ et $\mathrm{n}^{\circ} 229$. 
Mais il ne faut pas négliger le rôle des négociations conduites à la diète. Antonio da Besana s'y rend assidûment et expose les argumentations milanaises. Dans ces assemblées, le problème linguistique se pose certainement; bien que connu par plusieurs officiers suisses, le latin n'est pas utilisé dans les débats et les lettres du duc doivent être traduites en allemand ${ }^{26}$. Quelques Confédérés connaissaient probablement un peu d'italien car Antonio da Besana fait parfois appel à un interprète qu'il définit comme amigo ${ }^{27}$. Ce dernier traduit ses propos pendant ses interventions à la diète. Ayant la confiance de l'orateur, celui-ci est fort probablement un bourgeois de Lucerne dont la charge consiste à ce que les propos de l'orateur soient bien compris par les représentants des cantons. Cette aide linguistique semble cependant être limitée aux interventions officielles. L'absence d'autres références à un traducteur pousse à supposer que l'ambassadeur milanais possédait les bases de la langue allemande.

\subsection{Stratégies pour isoler la position du Canton d'Uri}

Une des difficultés majeures survenues durant les négociations avec les Suisses est la position du canton d'Uri qui exige que le nouveau traité inclue la cession formelle du territoire de la Vallée de la Léventine. Du fait que les opposants aux accords avec Milan soutiennent la position du waldstätte, Antonio da Besana doit mettre en place des stratégies aptes à remettre en cause la position uranaise. Son expérience et ses connaissances de la situation spécifique de cette vallée lui viennent en aide, tout comme le démontre les débats de janvier 1462.

Suite à l'énième requête des autorités d'Altdorf pour que le duché accepte cette annexion, l'orateur réagit en incluant dans les négociations la question du census que les hommes de la Léventine doivent encore au chapitre du Duomo de Milan (les documents en possession des chanoines parlent d'une somme de douze mille livres) ${ }^{28}$. Les accords conclus en

26. TD II, $\mathrm{n}^{\circ} 34$.

27. "In quest'hora è venuto uno amigo a mi, cioè quelo che è mio interprete a le volte quando io parlo in consilio.» (Ibid., $\mathrm{n}^{\circ}$ 1578). Il y a une certaine possibilité que cet amigo soit le marchand lucernois Anton Scherer (TD I, $\mathrm{n}^{\circ} 1563$ ).

28. «Finaliter pareno siano [i leventinesi] grandi debitori de li ordinarii de libre dodece mille, siché domino Christoforo Grasso ha producto una scriptura como la bona memoria del duca Philippo doveva luy proprio rescodere quisti denari, parendone.» (TD I, $\mathrm{n}^{\circ}$ 1478). 
1450 avec le duché imposent que ce type de querelle soit tranché par une procédure arbitrale conduite à Bellinzone ${ }^{29}$. Antonio da Besana se déplace donc dans cette ville avec une délégation composée de douze Suisses ${ }^{30}$. Là-bas, il est rejoint par Cristoforo Grassi, ordinaire du chapitre de l'Eglise majeure. A six reprises, les Confédérés sont sur le point d'abandonner les négociations et c'est seulement après de nombreuses disputes qu'on parvient à élire deux arbitres - Antonio da Besana en personne et le vicaire uranais de la Léventine - pour régler le problème. Les deux hommes revoient à la baisse les prétentions des chanoines et proposent le payement d'une somme de 2000 ducats ${ }^{31}$, le même chiffre que Filippo Maria Visconti avait promis de verser pour racheter les droits administratifs sur le territoire de la vallée.

Face aux obstructions uranaises dans les débats durant la diète, l'orateur milanais a donc poussé ses adversaires vers une problématique juridique qui ne touchait en rien aux relations diplomatiques (le différend est en effet entre le chapitre et les hommes de la vallée). Ainsi il a pu avancer sans trop de difficulté vers cet arbitrage qui met le duc de Milan, par le biais du chapitre du Duomo, dans la situation de pouvoir faire face aux conditions des accords de 1450 et - à la fin du mandat donné à Uri - de reprendre le contrôle sur la vallée ${ }^{32}$. Au vu de ces conclusions, les partisans du waldstätte abandonnent à toute vitesse les négociations et rentrent chez eux les mains vides et la bouche close ${ }^{33}$.

Un deuxième exemple de cette stratégie est mis en pratique par l'orateur et les partisans milanais à la diète en mai-juin 1466 pour faire face

29. «Iuxta la conventione et conclusione presa per voy ad nostro nome cum li magnifici signori Uraniesi de li confederati in la Liga de' Sviceri in la causa de li venerabili ordinari de la Chiesa Maiore de questa nostra cità de Milano contra quelli de la valle Leventina, como sapeti, nuy havemo ad mandare uno comissario ad Berinzona insieme con qualche veruno d'essi ordinari [...]. Però, havendovi ellecto voy, volimo ve trasferiati al dicto luocho de Berinzona.» (Ibid., nº 1467).

30. Ibid., $\mathrm{n}^{\circ} 1474$.

3I. Ibid., n ${ }^{\circ} 1486$.

32. Le duc de Milan suggère aussi de diviser le payement de ces tributs sur plusieurs années, afin de rappeler aux autorités d'Uri qu'ils administrent la vallée seulement en raison d'un traité qui va échouer: "Questo dicemo perché pratichando dicta conventione del futuro se non su li tri anni forse pigliaranno qualche ombrezza per rispecto del septennio che hanno ad tenire dicta valle quale si viene ad finire da qui ad tri anni.» (Ibid., $\mathrm{n}^{\circ}$ 1467).

33. Ibid., $\mathrm{n}^{\mathrm{o}} 1494$. 
aux poussés très peu diplomatiques d'un certain nombre de Confédérés qui désiraient profiter de la mort de Francesco Sforza pour imposer une véritable capitulation aux seigneurs de Milan. Bianca Maria Visconti et son fils Galeazzo Maria envisagent en effet la conclusion des négociations avec les Suisses et demandent aux Confédérés l'envoi d'une délégation plénipotentiaire ${ }^{34}$. Face à cette ouverture, les revendications du canton d'Uri surgissent en toute force et la grand majorité des délégués à la diète rencontre des difficultés à maintenir les négociations avec Antonio da Besana ${ }^{35}$. Les députés lui proposent enfin une stratégie: envoyer les délégués de cette minorité à Milan pour que leurs prétentions soient catégoriquement refusées par les nouveaux ducs ${ }^{36}$. A nouveau, le déplacement des négociations reflète un choix diplomatique bien précis qui vise à mettre en difficulté tous ceux qui veulent changer les équilibres géopolitiques et économiques entre la Confédération suisse et le duché de Milan.

34. "Volimo [nuy duci de Milano] in quello caso che, mandandose dicti oratori a nome della universale Liga, voi [Antonius da Besana] operiate et instate che li mandino con procura et sufficiente et amplo mandato de potere contrahere et concludere qui per ogni cosa, cioè tanto el facto della intelligentia come de Leventina." (TD II, $\mathrm{n}^{\circ} 38$ ).

35. "Et licet ad ogni vinti li decenove de tutti confederati piacesse meglio che qui se tractasseno et concludesseno le cose, dehinc se devenisse lí per la ratificatione et approbatiobe, nondimeno tante sono le inportune infestatione de li Uraniensi [...] che s'è concesso questa ambassaria a dicti Uraniensi ad le loro spexe, li quali fano et hano facto singulare pratica et instantia che se mandi lí, digando loro che 'l vostro stato è novo, tenero et debile et in timore de op<r>essione de' suoi inimici, siché facilmente in tale opportunità, mandando, se obtegniarà et Leventina et ogni altra cosa grata non tardando." (Ibid., n $\left.{ }^{\circ} 44\right)$.

36. Antonio da Besana décrit ainsi les agissements de la diète: «fu fata deliberatione che quisti ambasiatori non havesseno comissione né facultà de fare niuna cosa ape de' nostri illustrissimi signori, salvo de domandargli la reffirmatione de la exemptione deneralmente per tuto 'l dominio ducale et de domandare la Leventina et piú ultra in tuto, como voleno li Uraniensi, et che de fare confederatione o liga con prefati nostri signori non ne facesseno parola, et che in questo modo podevano concludere, aliter che retornasseno a casa \&c. [...]; item diceno che hano fato questo aciò che li Uraniensi, che inportunamente hano pur vogliuto questa ambasaria et non hano vogliuto, segondo el loro consciglio, havere patientia un poco per tractare su la amicitia et confederatione a comuna utilità de l'uno et de l'altro stato, non essendo exauditi da' nostri signori, retornano ad essi confederati et cognoscano l'erore comesso et havere mal fato in non fare al loro consciglio.» (Ibid., n 60). 


\subsection{Comprendre et connaitre les Suisses}

Au-delà des questions qui opposent les intérêts milanais aux aspirations des Confédérés, la plus grande difficulté à laquelle l'orateur milanais doit faire face reste les différentes appréciations du rôle de l'action politique: pour les standards milanais, la diplomatie suisse est toujours composée par des requêtes inopportunes, qui mènent trop souvent à des disputes et à des ruptures ${ }^{37}$. Pour les Confédérés, la diplomatie ne semble pas être un dialogue qui aboutit à un traité entre deux Etats, mais plutôt un marché par lequel il faut résoudre la totalité des querelles personnelles pendantes, gagner le maximum d'exemption et de droits et - bien-sûr recevoir un bon nombre de cadeaux et de bénéfices.

Néanmoins, l'action d'Antonio da Besana auprès des Confédérés ne se concentre pas seulement sur les négociations diplomatiques. A côté des discussions strictement liées aux débats menés à la diète, il existe une dimension sociale très importante que l'orateur doit prendre en compte. Il doit nécessairement connaître ses partenaires suisses non seulement sur le plan politique mais aussi par rapport à leurs intérêts, leurs ambitions, leur situation économique et leur entourage. Par ses contacts et ses connaissances au sein des communautés suisses, dont le noyau originel s'est très probablement formé lors de son expérience à Bellinzone, l'orateur a bien su mettre en place un véritable réseau qui lui garantit informations, opportunités et appuis à l'intérieur des institutions confédérées. Les chapitres précédents ont pu montrer quelques indices de cette situation, notamment dans la ville de Lucerne. Toutefois, elle est aussi très bien illustrée par les relations du représentant milanais avec quelques personnages spécifiques.

Un premier exemple est celui d'Anton Scherer, influent marchand lucernois qui poursuit pendant les négociations du Grande Capitolato une politique d'appui en faveur de l'Etat milanais, déjà commencée à l'époque de Filippo Maria Visconti ${ }^{38}$. L'existence probable d'une correspondance, dont il ne nous reste que des références secondaires et fragmentaires, souligne les liens entre cet homme et l'orateur. En 1463, il envoie à Antonio da Besana une lettre concernant les rapports entre

37. Antonio da Besana écrit ainsi au duc de Milan: «Stilo sempre a’ mei dí stilato da quisti è de fare simile inepte et quasi turpe requisitione.» (TD I, $\left.\mathrm{n}^{\circ} 1563\right)$.

38. Ibid., no 2076. 
Milan et les Suisses ${ }^{39}$, montrant avant tout le lien de confiance qui semble s'établir entre eux, mais aussi le fait que l'orateur joue un rôle d'intermédiaire même au-delà des missions diplomatiques spécifiques. En 1466, l'orateur intervient sur demande du duc auprès du podestà de Canobbio pour qu'Anton Scherer puisse obtenir justice pour des crédits qu'il tient dans la région ${ }^{40}$. A partir de ce lieu, après avoir en vain insisté, Antonio da Besana prend la route du Saint-Gothard avec le marchand suisse ${ }^{41}$. Enfin, les rapports - probablement même d'amitié - qui lient ces deux hommes sont aussi attestés par l'appui de l'orateur auprès des ducs de Milan pour que Scherer soit récompensé par d'importants cadeaux et même par une lettre de familiarité pour son aide aux négociations diplomatiques ${ }^{42}$.

Un cas similaire est celui de Heinrich Hansfurter, un politicien lucernois qui a travaillé aux côtés d'Antonio da Besana pour la médiation du Grande Capitolato ${ }^{43}$. A la différence d'Anton Scherer, très peu de documents nous informent sur les actions de ce personnage et les intérêts qu'il a pu entretenir avec l'orateur. Malgré ce manque de données, son apport doit être très important (ou du moins il doit en sembler ainsi à Milan) car en mars 1472, Galeazzo Maria conférera au lucernois - tout comme à Ham von Reschs de Zurich et à Peter von Wabern de Berne le titre de aulicus ducalis ${ }^{44}$.

Cependant, l'exemple le plus significatif de l'action de l'orateur auprès des Suisses est certainement l'évolution des rapports avec Heinrich von Hunwil, l'un des plus importants membres de l'élite lucernoise ${ }^{45}$. Ce personnage, déjà actif dans les pourparlers de 1454 , soutient très

39. Ibid., $\mathrm{n}^{\circ} 1525$ et $\mathrm{n}^{\circ} 1536$.

40. Plusieurs documents nous rapportent les difficultés du marchand lucernois dans cette querelle qui va perdurer sur toute la période 1464-1466. A la demande d'Anton Scherer, mais aussi par l'intermédiaire d'Antonio da Besana, le duc s'intéresse personnellement à la solution de cette dispute intervenant après du podestà et des débiteurs et demandant même qu'ils soient écoutés par le Conseil de Justice (Ibid., n ${ }^{\circ} 1509$, $\mathrm{n}^{\circ} 1616, \mathrm{n}^{\circ} 2099$ et passim).

4I. Ibid., no 2017 et no 2029.

42. TD II, $\mathrm{n}^{\circ} 514$ et $\mathrm{n}^{\circ} 515$.

43. Ibid., $\mathrm{n}^{\circ} 45, \mathrm{n}^{\circ} 415, \mathrm{n}^{\circ} 525$ et $\mathrm{n}^{\circ} 902$.

44. Ibid., $\mathrm{n}^{\circ} 1484$.

45. Heinrich von Hunwil (1418-1474) a été l'un des plus riches citoyens de Lucerne. Il est membre du Grand et du Petit conseil. Il tient la charge d'écuyer dans les années impaires 1449-1465, période dans laquelle il est aussi chargé de représenter la ville à la 
fortement les revendications du canton d'Uri et par ce biais reste très sceptique face aux propositions milanaises ${ }^{46}$. Dans un premier temps, Antonio da Besana exprime un jugement amer:

già sarea fata la conclusione [delle trattative] se quelo maladeto homo, Henrico Hunvil, non avesse seminato e non seminasse zizania ${ }^{47}$.

Toutefois en décembre 1469, l'orateur insiste auprès du duc pour que cet homme soit pardonné et soit inséré dans le nombre des partisans milanais à Lucerne, soulignant le fait qu'il a avec lui une "tanto optima rellatione» ${ }^{48}$. Ce changement d'opinion est étonnant et ne peut s'expliquer qu'en dehors du cadre diplomatique proprement dit, soit par des intérêts personnels, soit par un rapport d'amitié, soit par la pression du réseau lucernois qui pourrait avoir fortement insisté auprès de l'orateur pour réadmettre le politicien dans les grâces milanaises.

Enfin, l'exemple de Johann Herling du village de Sarnen montre que les liens entre Antonio da Besana et les Confédérés ne se restreignent pas qu'à son séjour à Lucerne. En effet, une pétition de cet homme auprès des seigneurs de Milan en 1477 nous informe qu'il a été au service de l'orateur pendant son séjour dans la ville lombarde ${ }^{49}$. L'absence d'une datation ne permet cependant pas l'approfondissement de cette circonstance et de son rapport - s'il y en a un - avec les missions diplomatiques de l'ambassadeur milanais.

\section{Jugement sur l'activité d'Antonio da Besana}

Les Confédérés ont bien apprécié l'action diplomatique d'Antonio de Besana: le respect pour l'orateur est exprimé à plusieurs reprises par les

diète confédérée (J. Kurmann, Die Politische Führungsschicht in Luzern 1450-1500, p. 69-70).

46. $T D$ I, $\mathrm{n}^{\circ} 475, \mathrm{n}^{\circ} 878 ; T D$ II, $\mathrm{n}^{\circ}$ 82. En 1466, en rentrant d'une ambassade infructueuse conduite pour la diète à Milan, il arrive même à proférer des mots injurieux contre les insignes ducaux, provoquant ainsi un ample incident diplomatique (TD II, $\mathrm{n}^{\mathrm{o}} 77$ ).

47. Ibid., no 82 .

48. Ibid., $\mathrm{n}^{\mathrm{o}} 1055$ et $\mathrm{n}^{\mathrm{o}} 1058$.

49. TD III, $\mathrm{n}^{\circ} 111$. 
autorités de la diète ${ }^{50}$ et même par certains personnages qui ne manquent pas d'en faire part au duc de Milan ${ }^{51}$. Du côté suisse, l'orateur est consideré «virum perfectum, rectum et pacis auctorem atque omnium bonorum utriusque status ac perpetue nostre confederationis amatorem pura veritate prepollentem " ${ }^{52}$. Les membres du Consiglio Secreto milanais sont du même avis, eux qui aimeraient confirmer l'ambassadeur, même contre l'avis de Galeazzo Maria Sforza, pour les missions qui ont lieu entre 1468 et $1472^{53}$.

D'autres contemporains milanais ont cependant une appréciation très différente de l'action d'Antonio da Besana. Bartolomeo Caìmi, podestà de Bellinzone entre 1462 et 1468 , cherche à plusieurs reprises à discréditer l'orateur auprès du duc. Lors des négociations de Bellinzone (1463), il écrit à Milan dénonçant la mauvaise conduite de l'ambassadeur:

Questo miser Antonio da Besana per li mali deportamenti fatti in Bellinzone per piú respecti luy non doveva componere né condurre tal composizione ${ }^{54}$.

Du même avis est Zanotto Visconti, commissaire des ducs de Milan à Bellinzone en 1466, pour qui Antonio da Besana n'est pas l'homme le plus apte à entretenir les relations diplomatiques avec les Confédérés 55 . Ces rivalités reflètent le changement dans l'appréciation des relations avec les Suisses qui advient au moment de la mort de Francesco Sforza: Bartolomeo Caími et Zanotto Visconti sont des hommes proches des nouveaux seigneurs de Milan et souhaiteraient une relève parmi les représentants diplomatiques.

Antonio da Besana est resté continuellement un officier de l'administration milanaise, lié à Francesco Sforza et très fortement appuyé par ce dernier. L'orateur a régi les négociations avec les Suisses pendant plus de dix ans. Toutefois au début de 1466, il n’a pas encore réussi à faire signer l'accord que Milan attend depuis longtemps. Il avait construit avec le duc un rapport d'extrême confiance qui lui a garanti une très large marge de manœuvre et qui lui a permis cet ajournement continu

50. $T D$ I, n $\mathrm{n}^{\circ} 1621 ; T D$ II, $\mathrm{n}^{\circ} 1041$.

5I. $T D$ II, $\mathrm{n}^{\circ} 45$.

52. Ibid., $\mathrm{n}^{\mathrm{o}} 1041$.

53. Ibid., $\mathrm{n}^{\mathrm{o}} 698, \mathrm{n}^{\mathrm{o}} 710, \mathrm{n}^{\mathrm{o}} 712, \mathrm{n}^{\mathrm{o}} 1330$ et $\mathrm{n}^{\circ} 1551$.

54. $T D \mathrm{I}, \mathrm{n}^{\circ} 1479$.

55. TD II, $\mathrm{n}^{\mathrm{o}} 12$ et $\mathrm{n}^{\mathrm{o}} 16$. 
des négociations. Ce rapport n'est pas le même avec le fils du duc qui, pressé par les jugements très contradictoires sur les actions de l'orateur, décide de lui confier d'autres tâches: celle de vicaire général (1468-1471), puis celle de maître des entrées extraordinaires (1472).

Le remplacement d'Antonio da Besana n'advint pas sans problèmes: son successeur Teodoro Piatti, qui fut choisi parmi les familles milanaises, n'avait qu'une connaissance académique du monde confédéré, des sujets des débats et des mœurs suisses. Sa mission tourne si mal que le duc de Milan fut contraint de rappeler l'ancien orateur pour renouer les contacts avec les Confédérés ${ }^{56}$. Ce tout dernier épisode conclut la période où l'orateur put donner sa contribution à la diplomatie septentrionale du duché de Milan.

\section{Conclusion}

Tout compte fait, les résultats obtenus par Antonio da Besana ne sont pas à la hauteur des attentes de la diplomatie milanaise: débats interminables, nombre excessif de concessions, très grandes dépenses financières. Cependant, par rapport à ses successeurs, ses ambassades ont toujours pu mettre un frein aux ambitions suisses au sud des Alpes: ses connaissances lui ont dicté une conduite prudente et très attentive aux conséquences pratiques des accords diplomatiques.

Antonio da Besana a toutefois su également appuyer ses propositions diplomatiques par une action dans le milieu social, phénomène exemplifié par ce groupe de partisans milanais présents dans la ville de Lucerne et aussi dans les autres cantons. Après l'éloignement d'Antonio da Besana, Galeazzo Maria Sforza chercha à prendre directement le contrôle de ce réseau, mais il n'obtint qu'un nombre considérable de lettres dans lesquelles les Suisses établirent la liste de leurs actions en faveur du duché de Milan et demandèrent récompenses et privilèges. En n'ayant plus d'intermédiaire qui comprenne exactement comment traiter avec les Confédérés et qui puisse organiser et mettre à profit ce réseau, le duché de Milan resta dans une impasse qui, aggravée par l'alliance du duché avec la Bourgogne, se concrétisera dans les échecs de toute négociation ultérieure pendant la seigneurie de Galeazzo Maria Sforza.

56. Ibid., $\mathrm{n}^{\mathrm{o}} 710$ et $\mathrm{n}^{\mathrm{o}} 712$. 
L'analyse de ce personnage tout à fait remarquable permet enfin de dresser le portrait d'une ambassade milanaise auprès de la Confédération suisse et de mettre en valeur quelques aspects plus généraux. Avant tout, le choix d'Antonio da Besana montre que le milieu dans lequel le duc choisit ses représentants est très vaste et comprend aussi les officiers qui travaillent loin de Milan. Ce choix met ensuite en évidence un profil très spécifique de compétences: études juridiques, expérience administrative, connaissance des sujets et des mours suisses: c'est à partir de ces points que se développe la forte confiance de Francesco Sforza en son représentant et par là la grande liberté d'action de l'orateur. Enfin, ces ambassades montrent l'importance du réseau social qui entoure Antonio da Besana dans les territoires suisses et qui lui permet de maîtriser et de poursuivre au mieux sa mission diplomatique.

Aureliano Martini

Université de Fribourg 


\section{BIBLIOGRAPHIE}

Cerioni, Lydia, La diplomazia sforzesca nella seconda metà del Quattrocento e i suoi cifrari segreti, Roma, Il centro di ricerca, 1970.

Chiesi, Giuseppe, Il Medioevo nelle carte: documenti di storia ticinese e svizzera dalle origini al secolo XVI, Bellinzona, Stato del Cantone Ticino, 1991.

—, "Venire cum equis ad partes Lumbardie: mercanti confederati nella seconda metà del XV secolo", Rivista Storica Svizzera, 44 (1994), p. $252-265$.

Chiesi, Giuseppe e Moroni Stampa, Luciano, Ticino Ducale, I: Francesco Sforza, Bellinzona, Stato del Cantone Ticino, 1993-1995.

-, Ticino Ducale, II : Galeazzo Maria Sforza, Bellinzona, Stato del Cantone Ticino, 1999-2003.

-, Ticino Ducale, III: Gian Galeazzo Maria Sforza, Bellinzona, Stato del Cantone Ticino, 2006.

Jucker, Michael, Gesandte, Schreiber, Akten: politische Kommunikation auf eidgenössischen Tagsatzungen im Spätmittelalter, Zürich, Chronos, 2004.

Kurmann, Josef, Die Politische Führungsschicht in Luzern 1450-1500, Luzern, Raeber, 1976.

Martini, Aureliano, "Dialogo e compromessi : trattative diplomatiche sulla via del San Gottardo", Bollettino Storico dello Svizzera Italiano, 111/1 (2008), p. 35-50.

Moroni Stampa, Luciano, "Francesco I Sforza e gli Svizzeri (14501466)", in Gli Sforza a Milano e in Lombardia e i loro rapporti co gli Stati italiani ed europei (1450-1535). Convegno internazionale, Milano, 18-21 maggio 1981, Milano, Cisalpino-Goliardica, 1982, p. 599-608.

Vaglienti, Francesca M., "Per dicta pace realegrati”: le trattative diplomatiche tra la Confederazione Elvetica e il duca Galeazzo 
M. Sforza per il rinnovo del Capitolato, l'investitura della Leventina e la cessione della Val Formazza (1466-1469)», Archivio Storico Ticinese, 31/116 (1994), p. 125-166. 